\title{
Transcriptional similarity in couples reveals the impact of shared environment and lifestyle on gene regulation through modified cytosines
}

Ke Tang, Wei Zhang

Gene expression is a complex and quantitative trait that is influenced by both genetic and non-genetic regulators including environmental factors. Evaluating the contribution of environment to gene expression regulation and identifying which genes are more likely to be influenced by environmental factors are important for understanding human complex traits. We hypothesize that by living together as couples, there can be commonly coregulated genes that may reflect the shared living environment (e.g., diet, indoor air pollutants, behavioral lifestyle). The lymphoblastoid cell lines (LCLs) derived from unrelated couples of African ancestry (YRI, Yoruba people from Ibadan, Nigeria) from the International HapMap Project provided a unique model for us to characterize gene expression pattern in couples by comparing gene expression levels between husbands and wives. Strikingly, 778 genes were found to show much smaller variances in couples than random pairs of individuals at a false discovery rate (FDR) of $5 \%$. Since genetic variation between unrelated family members in a general population is expected to be the same assuming a random-mating society, non-genetic factors (e.g., epigenetic systems) are more likely to be the mediators for the observed transcriptional similarity in couples. We thus evaluated the contribution of modified cytosines to those genes showing transcriptional similarity in couples as well as the relationships these CpG sites with other gene regulatory elements, such as transcription factor binding sites (TFBS). Our findings suggested that transcriptional similarity in couples likely reflected shared common environment partially mediated through cytosine modifications. 
1 Transcriptional Similarity in Couples Reveals the Impact of Shared Environment and

2

3

\section{Lifestyle on Gene Regulation through Modified Cytosines}

Ke Tang ${ }^{2}$ and Wei Zhang ${ }^{1,3 *}$

$4 \quad{ }^{1}$ Insitute of Precision Medicine, Jining Medical University, Jining 272067, China

5 2Department of Bioengineering, University of Illinois, Chicago, IL 60612, USA

6 3 ${ }^{3}$ epartment of Preventive Medicine, Northwestern University Feinberg School of Medicine,

7 Chicago, IL 60611, USA

8

$9{ }^{*}$ Correspondence to: Wei Zhang, Ph.D., 680 N. Lake Shore Drive, Suite 1400, Chicago, IL

10 60611, USA; Tel: +1 (312) 503-1040; Fax: 312-908-9588; E-mail:

11 wei.zhang1@,northwestern.edu

12

13

14 Running head: Gene expression in couples 


\section{ABSTRACT}

26 Gene expression is a complex and quantitative trait that is influenced by both genetic and non-

27 genetic regulators including environmental factors. Evaluating the contribution of environment

to gene expression regulation and identifying which genes are more likely to be influenced by

29 environmental factors are important for understanding human complex traits. We hypothesize

that by living together as couples, there can be commonly co-regulated genes that may reflect the

31 shared living environment (e.g., diet, indoor air pollutants, behavioral lifestyle). The

32 lymphoblastoid cell lines (LCLs) derived from unrelated couples of African ancestry (YRI,

33 Yoruba people from Ibadan, Nigeria) from the International HapMap Project provided a unique

34 model for us to characterize gene expression pattern in couples by comparing gene expression

35 levels between husbands and wives. Strikingly, 778 genes were found to show much smaller

36 variances in couples than random pairs of individuals at a false discovery rate (FDR) of 5\%.

37 Since genetic variation between unrelated family members in a general population is expected to

38 be the same assuming a random-mating society, non-genetic factors (e.g., epigenetic systems) are

39 more likely to be the mediators for the observed transcriptional similarity in couples. We thus

40 evaluated the contribution of modified cytosines to those genes showing transcriptional

41 similarity in couples as well as the relationships these $\mathrm{CpG}$ sites with other gene regulatory

42 elements, such as transcription factor binding sites (TFBS). Our findings suggested that

43 transcriptional similarity in couples likely reflected shared common environment partially

44 mediated through cytosine modifications. 


\section{RESEARCH HIGHLIGHTS}

47 We compared gene expression levels in couples and random pairs of individuals in a well-

48 characterized collection of HapMap samples. A substantial number of genes showed higher

49 similarity in couples than in random pairs of males and females. Cytosine modifications

50 accounted partially for the observed gene expression similarity in couples. Certain

51 transcription factor binding sites were found to be enriched in genes more similar in couples.

53 Key Words: Lymphoblastoid cell line; HapMap; Gene expression; Epigenetics; Cytosine

54 modification; Transcription factor binding site 
Gene expression is a complex quantitative trait that may be influenced by both genetic

66

67

68

69

and non-genetic factors, such as environment. Besides genetic variants identified as eQTL (expression quantitative trait loci), more recently, contributions from epigenetic systems, such as microRNAs, modified cytosines, and histone modifications to gene expression phenotypes have been investigated in various studies including those using the International HapMap Project (HapMap, 2003; HapMap, 2005) lymphoblastoid cell lines (LCLs) derived from apparently healthy individuals (Huang et al., 2011; McVicker et al., 2013; Moen et al., 2013; Zhang et al., 2014). In addition, substantial gene-environment interactions have begun to be demonstrated in gene expression studies including a recent study based on transcriptomic sequencing assay in twins (Buil et al., 2015). Characterizing which genes are more likely to be influenced by environmental factors (e.g., shared living environment, behavioral lifestyle) as well as their relationships with genetic and epigenetic variations can enhance our understanding of human complex traits, given the fundamental roles of gene expression in determining traits and phenotypes.

$$
\text { Specifically, in this work, we utilized the unrelated couples from the HapMap YRI }
$$

(Yoruba people from Ibadan, Nigeria) LCL panel to characterize gene expression pattern in couples, taking advantage of the whole-genome gene expression data that have been profiled from our previous publication using the Affymetrix Human Exon 1.0 ST Array (exon array) (Zhang et al., 2008). Genes showing the 'couple effect' of regulation (i.e., transcriptional similarity between husbands and wives) may reflect the shared common living environment and behavioral lifestyle in couples, who should be genetically independent in a random-mating society. To further evaluate the potential contribution by modified cytosines (i.e., methylation at CpG dinucleotides) to the 'couple effect' of regulation, we integrated our previously published 
88 cytosine modification data on these samples using the Illumina HumanMethylation450 BeadChip

89 (450K array) (Moen et al., 2013). This work aims to shed novel light into clinical observations

90 on spousal correlations of lifestyle-related risk factors for diseases (e.g., cardiovascular diseases)

91 (Jurj et al., 2006; Di Castelnuovo et al., 2009), as well as implicate cytosine modifications as a

92 critical epigenetic gene regulation mechanism that may mediate the shared environment and

93 lifestyle.

95 MATERIALS AND METHODS

96 The workflow, data analysis and thresholds used are summarized in Figure 1.

97 Detection of genes showing the 'couple effect' of regulation

98 Whole-genome gene expression data (GSE7851) on a collection of HapMap YRI LCL samples

99 were previously generated using the Affymetrix Human Exon 1.0ST Array (exon array) (Zhang

100 et al., 2008). Sample preparation, array profiling, data processing, summarization, and

101 normalization were described in our previous publication (Zhang et al., 2008). Selected genes

102 from the exon array data have been experimentally validated (Zhang et al., 2008; Zhang et al.,

103 2009)._In total, 14591 gene-level transcript clusters that were mapped to unique Entrez Gene IDs

104 in 29 unrelated YRI couples (58 individuals) were used for testing the 'couple effect' of gene

105 regulation. The 'couple effect' was measured by the difference of gene expression levels in a

106 couple of sample $A$ and sample $B$ : 
$d_{i}^{A, B}=\frac{\left|x_{i}^{A}-x_{i}^{B}\right|}{\widetilde{x}_{i}}$, where $x_{i}^{A}$ and $x_{i}^{B}$ are the expression levels of gene $i$ in sample $A$ and $B$,

108 separately. $\widetilde{x}_{i}$ is the median of expression for gene $i$. To control false discovery rate (FDR), we

109 calculated empirical p-values by performed 10000 random samplings in these samples. In each

110 sampling, we randomly assembled 29 pairs of males and females from the 58 individuals without

111 replacement. The order of samplings was retained for all of the tested genes. For each gene, a

112 one-tailed t-test was used to determine if the difference of gene expression in couples was

113 smaller than that in the same number of random pairs. We also calculated Pearson correlations of

114 gene expression differences between real couples and randomly assembled non-family male-

115 female pairs.

116 Linking modified cytosines to genes with the 'couple effect' of regulation

117 Cytosine modification data (GSE39672) were previously profiled by us using the Illumina

118 Infinium HumanMethylation450 BeadChip platform (450K array) (Moen et al., 2013). DNA

119 sample preparation, 450K array profiling, data processing, summarization and normalization

120 were described in our previous publication (Moen et al., 2013). Selected CpGs from the cytosine

121 modification dataset have been experimentally validated using bisulfite sequencing (Moen et al.,

122 2013). A total of 283,540 autosomal CpG sites (Zhang et al., 2012) after removing CpG sites

123 ambiguously mapped to the human genome and CpG sites containing common SNPs were

124 included in the current study. We tested for correlation between gene expression levels and the

125 M-values (Du et al., 2010) of local CpG probes, defined as CpG sites located within the 10kb

126 regions upstream of the transcription start sites (TSS) or downstream of the transcription end

127 sites (TES) based on the RefSeq database hg19 (Pruitt et al., 2014). 
129 We used the DAVID (Database for Annotation, Visualization and Integrated Discovery) tool

130 (Huang da et al., 2009b; Huang da et al., 2009a) to systematically search if there were any

131 functional terms, such as Gene Ontology (GO) (Ashburner et al., 2000) biological processes and

132 motifs (e.g., TFBS, transcription factor binding sites) enriched among the identified genes with

133 the 'couple effect' of regulation relative to the human genome reference at a Benjamini adjusted

134 p-value of $1 \%$.

136 RESULTS

137 Transcriptional similarity in couples

138 Overall, based on the 14591 analyzed genes, the correlations between male and female samples

139 in couples are higher than non-family male-female pairs in the YRI samples (Figure 2a). The

140 peak of the correlation curve of true couples tended to shift towards the higher correlation values

141 compared to non-family male-female pairs. In total, 778 gene-level transcript clusters showed

142 significantly smaller variance couples than in random pairs of males and females at an empirical

143 p-value $<0.05$ (Supplemental Table S1). The distribution of empirical p-values (Figure 2b)

144 showed an obvious bias towards genes with smaller variance in couples. For these 778 genes

145 with the 'couple effect' of regulation, the correlations between male and female samples in true

146 couples were significantly higher than non-family male-female pairs (Figure $2 \mathrm{c}$ ). The mean and

147 median of correlations in true couples were 0.984 and 0.986 , versus 0.971 and 0.972 in non-

148 family male-female pairs. The overlapped area under the curve (AUC) is 0.22 , indicating that 
149 gene expression levels of a substantial number of genes in couples are more similar compared to

150 random pairs of individuals.

151 Linking modified cytosines with genes showing the 'couple effect' of regulation

152 In total, 16129 local $\mathrm{CpG}$ sites (i.e., within10kb up- and down-stream of genes) were annotated

153 to the 778 genes with the 'couple effect' of regulation. These CpG sites can be grouped into three

154 major categories: upstream (-10kb to TSS) (3404 CpGs), gene body (9261 CpGs), and

155 downstream (TES to $+10 \mathrm{~kb})(3464 \mathrm{CpGs})$. At q-value $<0.05$, seven local $\mathrm{CpG}$ sites were found to

156 be associated with six genes in the YRI samples: EMIDl (encoding EMI domain containing 1),

157 SNRK (encoding SNF related kinase), MAPKSP1 (encoding MAPK Scaffold Protein 1), DPYSL2

158 (dihydropyrimidinase-like 2), TCN2 (encoding transcobalamin II) and ZCCHC14 (encoding zinc

159 finger, $\mathrm{CCHC}$ domain containing 14) (Table 1). For example, the modification levels of CpG site

$160 \operatorname{cg} 24811472$, located in the gene body, were positively associated with the expression levels of

161 DPYSL2 (Figure 3a). Among these six CpG-regulating genes, DPYSL2 is known to be associated

162 with schizophrenia and bipolar disorder (Fallin et al., 2005); while TCN2 is associated with

163 various disorders including Alzeimer's disease, vascular disease, and certain cancers (e.g., brain

164 and colorectal) (Hazra et al., 2010).

165 Functional annotation analysis

166 We searched for enriched functional annotations among the 778 genes with the 'couple effect' of

167 regulation in the YRI samples. Notably, 64 TFBS (Supplemental Table S2) were detected to be

168 associated with these genes at a Benjamini adjusted $\mathrm{p}$-value $<1 \%$. Among them, the exon array

169 data of 29 transcription factors (TF) are available for testing associations between local CpGs

170 and gene expression of TF. A number of local CpGs were significantly associated with the TF 
171 gene expression at $\mathrm{p}<0.05$ (Table S3). For instance, the modification levels of CpG site

$172 \operatorname{cg} 19750321$ were negatively associated with ARNT (encoding aryl hydrocarbon receptor nuclear

173 translocator) expression in the YRI samples (Figure 3b). These results indicated a potential route

174 of regulation, in which local TF CpGs may be influenced by environment; TF CpGs may

175 regulate their corresponding TF gene expression; then TF regulate target gene expression. In

176 addition, 19 out the 778 genes are located on chromosome 19p13.3, representing an enrichment

177 of 4.2-fold relative to the human genome reference (Bemjamini adjusted $p=4.5 \mathrm{E}-4$ ).

178 Interestingly, $L D L R$ (encoding low density lipoprotein receptor), located on chromosome

$17919 \mathrm{p} 13.3$ is known to be associated with various diseases, such as coronary artery disease and

180 dyslipidemia (Martinelli et al., 2010). Couple concordance has been found in coronary artery

181 disease. The total serum cholesterol and low-density lipoprotein (LDL) cholesterol were

182 significantly lower for the wives whose husbands exposed to a continuous coronary heart disease 183 risk-factor intervention program (Sexton et al., 1987).

\section{DISCUSSION}

187 In this paper, our findings demonstrated that a substantial number of genes showed smaller 188 variances in couples than random pairs of males and females. A link between cytosine

189 modifications to some of these genes with the 'couple effect' of regulation suggested that

190 modified cytosines contributed partially to this gene expression pattern in couples, thus likely

191 mediating the influence of shared living environment and behavioral lifestyle on gene expression 192 among family members. 
193 Elucidating environmental effect on gene expression regulation will enhance our understanding

194 of complex traits and diseases. Clinically, spousal concurrences of certain diseases are not

195 uncommon. For example, people are found at increased risk of having asthma, hypertension,

196 hyperlipidaemia and pepticulcer diseases when their spouses have these diseases (Hippisley-Cox

197 et al., 2002). In this study, taking advantage of the HapMap LCL samples, on which we have

198 accumulated a tremendous resource of gene expression and epigenomic data (Zhang et al., 2013),

199 we explored to identify a substantial number of genes showing the 'couple effect' of regulation

200 (i.e., transcriptional similarity in couples) in the YRI samples derived from African individuals.

201 Though overall, there were no significant enrichment of GO biological processes or canonical

202 pathways among the 778 genes with the 'couple effect' of regulation, some of these genes are

203 known to be associated with certain traits and diseases that have been shown to be familial.

204 Notably, chromosome 19p13.3, which contains 19 genes showing the 'couple effect' of

205 regulation, was enriched relative to the human genome. Genes on chromome 19p13.3 have been

206 associated with various diseases. In particular, $L D L R$, a gene showing the 'couple effect' of

207 regulation in this study, has been associated with familial hypercholesterolemia and coronary

208 artery disease (Martinelli et al., 2010). In a previous study, couple concordance was found in

209 coronary artery disease as the total low-density lipoprotein (LDL) cholesterol and serum

210 cholesterol were significantly lower for the wives whose husbands exposed to a continuous

211 coronary heart disease risk-factor intervention program compared to the wives from control

212 group (Sexton et al., 1987). Our findings, therefore, suggested a potential link between shared

213 living environment and behavioral lifestyle and the expression patterns of a number of genes,

214 which in turn may explain spousal or familial concurrences of certain diseases and phenotypes. 
215 We further evaluated whether epigenetic systems, specifically cytosine modifications might

216 contribute to the observed 'couple effect' of regulation. Modification levels of local CpG sites

217 were found to account for several genes that showed the 'couple effect' of regulation. Among

218 these CpG-regulated genes (Table 1), a few are known to be associated with complex diseases.

219 For example, two local $\mathrm{CpG}$ sites in body regions were associated with the expression levels of

220 DPYSL2, which is known to be associated with psychiatric disorders including schizophrenia and

221 bipolar disorder. In another example, one local $\mathrm{CpG}$ site in body region was associated with the

222 expression of $T C N 2$, which is associated with Alzeimer's disease and certain cancers including

223 colorectal cancer (Hazra et al., 2010) . Interestingly, our results indicated a possible link of

224 shared living environment and behavioral lifestyle in the regulation of TCN2 that belongs to the

225 one-carbon metabolism pathway, which has been associated with the risk for colorectal cancer.

226 Since in a random-mating society, genetic background in couples is independent, $\mathrm{CpG}$

227 modifications, therefore likely function as the mediators for the environmental effect on gene

228 expression in a shared living environment. In addition to association of local $\mathrm{CpG}$ sites and some

229 genes with the 'couple effect' of regulation, there were also a few cases in which $\mathrm{CpG}$

230 modifications mediating environmental factors through regulating transcription factors, which in

231 turn may regulate the target genes that showed the 'couple effect' of regulation.

232 In this study, we used couples from the HapMap YRI panel to represent shared

233 environment for a significant length of time (i.e., long enough to have children). It may not be as

234 ideal as using a twin design to dissect environmental factors from genetic factors, but using these

235 HapMap couple data allowed us to explore directly how epigenetic systems may mediate the

236 shared living environment and behavioral lifestyle in regulating gene expression in a well-

237 characterized collection of samples, on which both gene expression and cytosine modification 
238 data are available. Other potential limitations may include the lack of age information on the

239 HapMap samples, given that age is a likely factor affecting DNA methylation. Our findings

240 warrant future more comprehensive investigations that integrate other critical epigenetic systems

241 such as histone modifications to elucidate the 'couple effect' of gene regulation, which could

242 improve our understanding of the complex relationships between environmental factors (e.g.,

243 lifesyles, behavior, air pollution, diet) and health conditions.

\section{References}

250

251

252

253

254

255

256

257

258

259

260

261

262

263

264

Ashburner, M., Ball, C.A., Blake, J.A., Botstein, D., Butler, H., Cherry, J.M., Davis, A.P., Dolinski, K., Dwight, S.S., Eppig, J.T., Harris, M.A., Hill, D.P., Issel-Tarver, L., Kasarskis, A., Lewis, S., Matese, J.C., Richardson, J.E., Ringwald, M., Rubin, G.M. and Sherlock, G., 2000. Gene ontology: tool for the unification of biology. The Gene Ontology Consortium. Nat Genet 25, 25-9.

Buil, A., Brown, A.A., Lappalainen, T., Vinuela, A., Davies, M.N., Zheng, H.F., Richards, J.B., Glass, D., Small, K.S., Durbin, R., Spector, T.D. and Dermitzakis, E.T., 2015. Gene-gene and gene-environment interactions detected by transcriptome sequence analysis in twins. Nat Genet 47, 88-91.

Di Castelnuovo, A., Quacquaruccio, G., Donati, M.B., de Gaetano, G. and Iacoviello, L., 2009. Spousal concordance for major coronary risk factors: a systematic review and metaanalysis. Am J Epidemiol 169, 1-8.

Du, P., Zhang, X., Huang, C.C., Jafari, N., Kibbe, W.A., Hou, L. and Lin, S.M., 2010. Comparison of Beta-value and $\mathrm{M}$-value methods for quantifying methylation levels by microarray analysis. BMC Bioinformatics 11, 587. 
265

Fallin, M.D., Lasseter, V.K., Avramopoulos, D., Nicodemus, K.K., Wolyniec, P.S., McGrath, J.A., Steel, G., Nestadt, G., Liang, K.Y., Huganir, R.L., Valle, D. and Pulver, A.E., 2005. Bipolar I disorder and schizophrenia: a 440-single-nucleotide polymorphism screen of 64 candidate genes among Ashkenazi Jewish case-parent trios. Am J Hum Genet 77, 918-36.

HapMap, 2003. The International HapMap Project. Nature 426, 789-96.

HapMap, 2005. A haplotype map of the human genome. Nature 437, 1299-320.

Hazra, A., Fuchs, C.S., Kawasaki, T., Kirkner, G.J., Hunter, D.J. and Ogino, S., 2010. Germline polymorphisms in the one-carbon metabolism pathway and DNA methylation in colorectal cancer. Cancer Causes Control 21, 331-45.

Huang da, W., Sherman, B.T. and Lempicki, R.A., 2009a. Bioinformatics enrichment tools: paths toward the comprehensive functional analysis of large gene lists. Nucleic Acids Res $37,1-13$.

Huang da, W., Sherman, B.T. and Lempicki, R.A., 2009b. Systematic and integrative analysis of large gene lists using DAVID bioinformatics resources. Nat Protoc 4, 44-57.

Huang, R.S., Gamazon, E.R., Ziliak, D., Wen, Y., Im, H.K., Zhang, W., Wing, C., Duan, S., Bleibel, W.K., Cox, N.J. and Dolan, M.E., 2011. Population differences in microRNA expression and biological implications. RNA Biol 8, 692-701.

Jurj, A.L., Wen, W., Li, H.L., Zheng, W., Yang, G., Xiang, Y.B., Gao, Y.T. and Shu, X.O., 2006. Spousal correlations for lifestyle factors and selected diseases in Chinese couples. Ann Epidemiol 16, 285-91.

Martinelli, N., Girelli, D., Lunghi, B., Pinotti, M., Marchetti, G., Malerba, G., Pignatti, P.F., Corrocher, R., Olivieri, O. and Bernardi, F., 2010. Polymorphisms at LDLR locus may be associated with coronary artery disease through modulation of coagulation factor VIII activity and independently from lipid profile. Blood 116, 5688-97.

McVicker, G., van de Geijn, B., Degner, J.F., Cain, C.E., Banovich, N.E., Raj, A., Lewellen, N., Myrthil, M., Gilad, Y. and Pritchard, J.K., 2013. Identification of genetic variants that affect histone modifications in human cells. Science 342, 747-9.

Moen, E.L., Zhang, X., Mu, W., Delaney, S.M., Wing, C., McQuade, J., Myers, J., Godley, L.A., Dolan, M.E. and Zhang, W., 2013. Genome-wide variation of cytosine modifications between European and African populations and the implications for complex traits. Genetics 194, 987-96.

Pruitt, K.D., Brown, G.R., Hiatt, S.M., Thibaud-Nissen, F., Astashyn, A., Ermolaeva, O., Farrell, C.M., Hart, J., Landrum, M.J., McGarvey, K.M., Murphy, M.R., O'Leary, N.A., Pujar, S., Rajput, B., Rangwala, S.H., Riddick, L.D., Shkeda, A., Sun, H., Tamez, P., Tully, R.E., Wallin, C., Webb, D., Weber, J., Wu, W., DiCuccio, M., Kitts, P., Maglott, D.R., Murphy, T.D. and Ostell, J.M., 2014. RefSeq: an update on mammalian reference sequences. Nucleic Acids Res 42, D756-63. 
302 Sexton, M., Bross, D., Hebel, J.R., Schumann, B.C., Gerace, T.A., Lasser, N. and Wright, N.,

303

304

305

306

307

308

309

310

311

312

313

314

315

316

317

318

319

320

321

322

323

324

325

326

327

328

329

330

331

332

333

334

1987. Risk-factor changes in wives with husbands at high risk of coronary heart disease (CHD): the spin-off effect. J Behav Med 10, 251-61.

Zhang, W., Duan, S., Bleibel, W.K., Wisel, S.A., Huang, R.S., Wu, X., He, L., Clark, T.A., Chen, T.X., Schweitzer, A.C., Blume, J.E., Dolan, M.E. and Cox, N.J., 2009. Identification of common genetic variants that account for transcript isoform variation between human populations. Hum Genet 125, 81-93.

Zhang, W., Duan, S., Kistner, E.O., Bleibel, W.K., Huang, R.S., Clark, T.A., Chen, T.X., Schweitzer, A.C., Blume, J.E., Cox, N.J. and Dolan, M.E., 2008. Evaluation of genetic variation contributing to differences in gene expression between populations. Am J Hum Genet 82, 631-40.

Zhang, W., Zheng, Y. and Hou, L., 2013. Pharmacogenomic Discovery Delineating the Genetic Basis of Drug Response. Curr Genet Med Rep 1, 143-149.

Zhang, X., Moen, E.L., Liu, C., Mu, W., Gamazon, E.R., Delaney, S.M., Wing, C., Godley, L.A., Dolan, M.E. and Zhang, W., 2014. Linking the genetic architecture of cytosine modifications with human complex traits. Hum Mol Genet 23, 5893-905.

Zhang, X., Mu, W. and Zhang, W., 2012. On the analysis of the illumina 450k array data: probes ambiguously mapped to the human genome. Front Genet 3, 73.

Table 1. CpG-Gene expression associations at q-value $<0.05$ between genes with the 'couple effect' and their local CpGs.

\begin{tabular}{|l|l|r|r|r|r|r|}
\hline CpGID & Gene Symbol & \multicolumn{1}{l|}{$\begin{array}{l}\text { Affymetrix } \\
\text { ID }\end{array}$} & CpGvalue & p-value & q-value & $r$ \\
\hline cg02152034 & EMID1 & 3941848 & -0.3732414 & $1.42 \mathrm{E}-06$ & 0.0227167 & -0.602693 \\
\hline cg10527635 & SNRK & 2619666 & -0.4224054 & $6.71 \mathrm{E}-06$ & 0.04227625 & -0.570362 \\
\hline cg12001078 & MAPKSP1 & 2779408 & -0.2527613 & $8.37 \mathrm{E}-06$ & 0.04227625 & -0.565482 \\
\hline cg24811472 & DPYSL2 & 3091077 & 0.9650537 & $1.06 \mathrm{E}-05$ & 0.04227625 & 0.560246 \\
\hline cg17759595 & DPYSL2 & 3091077 & 0.5309326 & $1.33 \mathrm{E}-05$ & 0.04262027 & 0.554953 \\
\hline cg04081402 & TCN2 & 3942472 & -0.5538604 & $1.70 \mathrm{E}-05$ & 0.04535223 & -0.549258 \\
\hline
\end{tabular}




\begin{tabular}{|l|l|l|l|l|l|l|}
\hline cg06545761 & ZCCHC14 & 3703665 & 0.0759574 & $1.99 \mathrm{E}-05$ & 0.04554208 & 0.545513 \\
\hline
\end{tabular}

335

336

337

338

339

340

341

342

343

344

345

346

347

348

349

350

351

352

353

Figure Legends

354

355 Figure 1. An overview of the workflow.

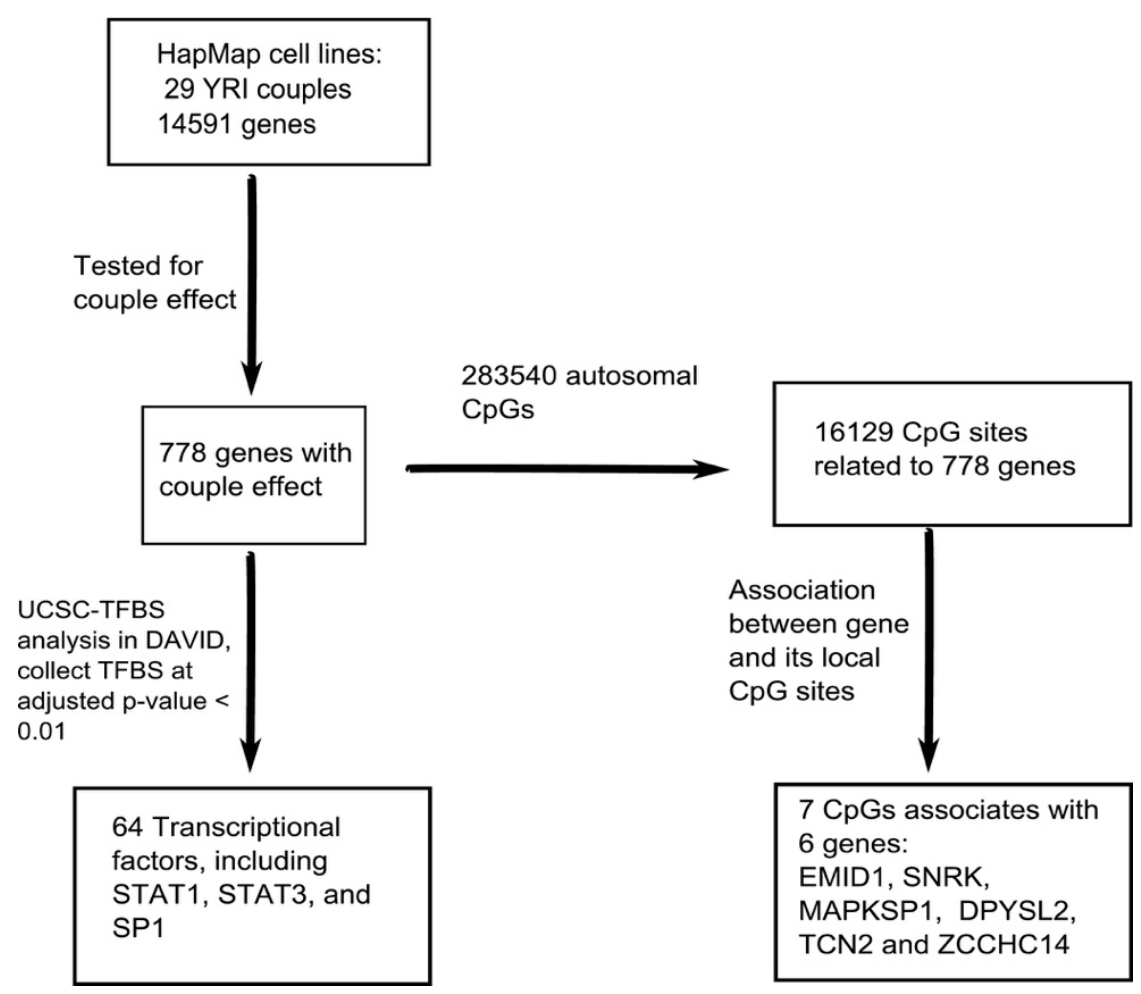


357 In total, 14591 genes were tested for the couple effect in 29 Yoruba couples. 778 genes were

358 detected at empirical p-value $<0.05$. NIH/DAVID functional analysis showed 64 TFBS

359 (transcription factor binding sites) enriched among these genes at FDR $<0.01$. Among the total

360283540 autosomal CpGs, $16129 \mathrm{CpGs}$ are annotated to the 778 genes. Seven cis-acting

361 associations were found between these genes and their local CpGs.

362

363

364

365 Figure 2. The couple effect of genetic expression.

a

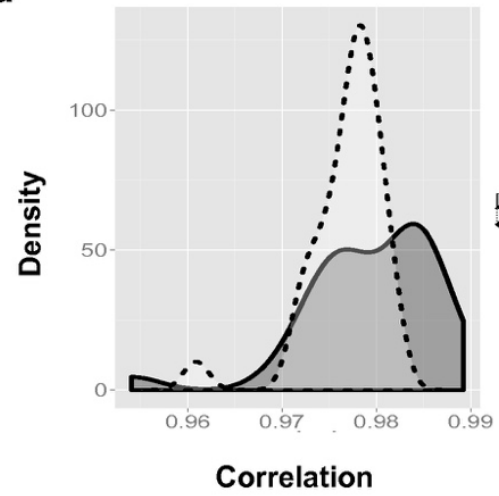

C

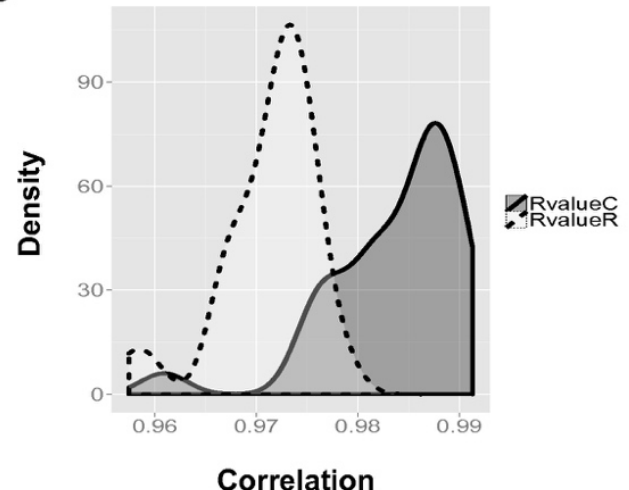

b

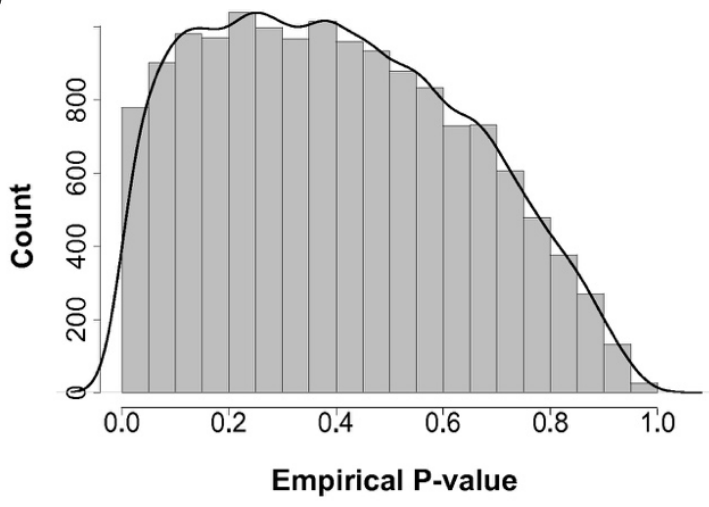

d

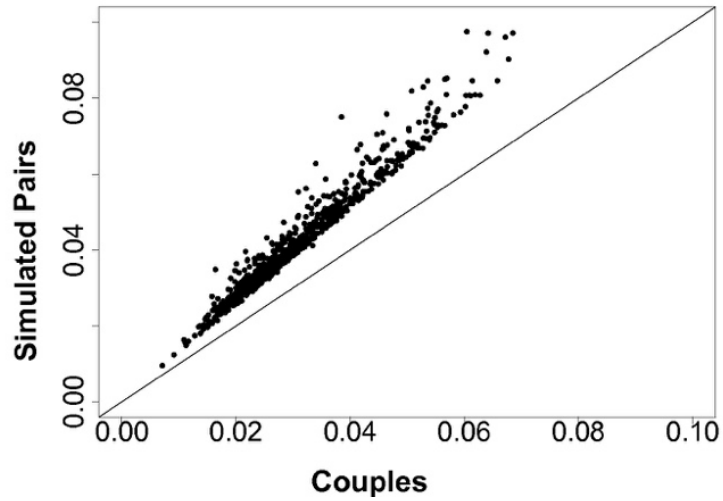


367 (a) The density plot of correlations between male and female samples in true couples (RvalueC) 368 and non-family male-female pairs (RvalueR) in all 14591 genes. (b) The distribution of empirical

369 p-values of couple effect of gene expression. (c) The density plot of correlations between male

370 and female samples in true couples (RvalueC) and non-family male-female pairs (RvalueR) in

371 the 778 genes at empirical p-values $<0.05$. (d) Differences between male and female samples in

372 gene expression. For genes with couple effect detected at 5\% empirical p-value, the average

373 differences between male and female samples from simulated (Y-axis) were compared with the

374 male-female differences from true couples (X-axis).

377 Figure 3. Examples showing that cytosine modifications account for the 'couple effect' of 378 gene expression.

a

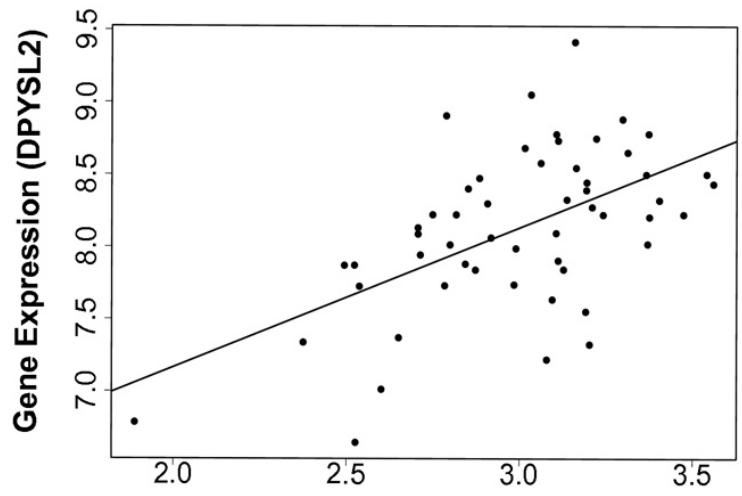

Modification Level M-value (cg24811472) chr8

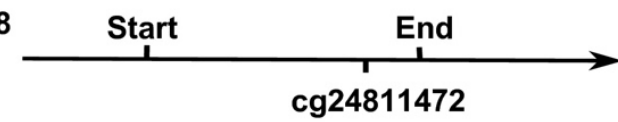

b
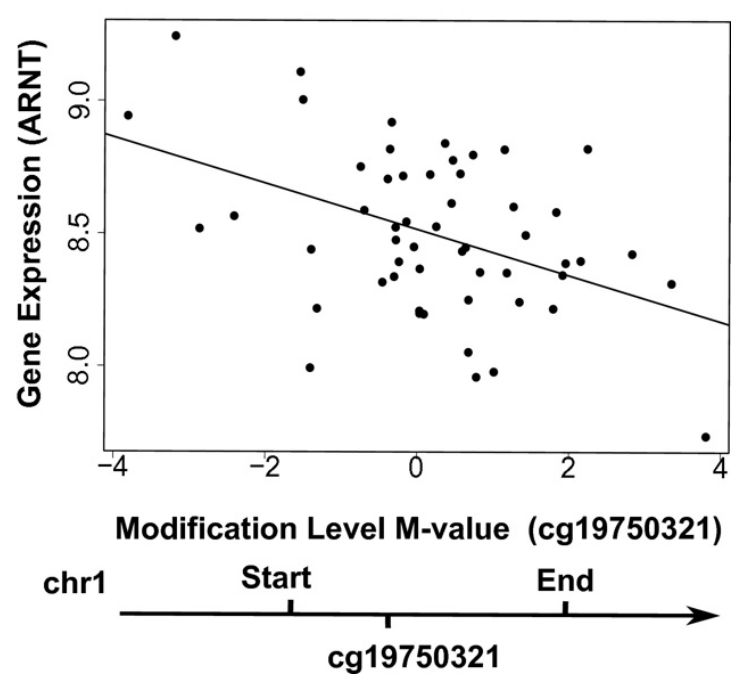

(a) Gene expression level of DPYSL2 (encoding dihydropyrimidinase-like 2) is significantly associated with a gene-body CpG site $\operatorname{cg} 24811472$ (p=1.06E-05). (b) Gene expression level of 
$382 A R N T$ (encoding aryl hydrocarbon receptor nuclear translocator) is significantly associated with

383 a gene-body CpG site $\operatorname{cg} 19750321(\mathrm{p}=0.001)$.

384 


\section{1}

An overview of the workflow

In total, 14591 genes were tested for the couple effect in 29 Yoruba couples. 778 genes were detected at empirical p-value $<0.05$. NIH/DAVID functional analysis showed 64 TFBS (transcription factor binding sites) enriched among these genes at FDR $<0.01$. Among the total 283540 autosomal CpGs, 16129 CpGs are annotated to the 778 genes. Seven cis-acting associations were found between these genes and their local CpGs. 
HapMap cell lines:

29 YRI couples

14591 genes

Tested for couple effect

UCSC-TFBS

analysis in DAVID, collect TFBS at adjusted $p$-value < 0.01

64 Transcriptional factors, including STAT1, STAT3, and SP1
283540 autosomal CpGs
16129 CpG sites related to 778 genes

Association between gene and its local CpG sites

7 CpGs associates with 6 genes:

EMID1, SNRK, MAPKSP1, DPYSL2, TCN2 and $\mathrm{ZCCHC} 14$ 


\section{2}

The couple effect of genetic expression.

(a) The density plot of correlations between male and female samples in true couples

(RvalueC) and non-family male-female pairs (RvalueR) in all 14591 genes. (b) The distribution of empirical p-values of couple effect of gene expression. (c) The density plot of correlations between male and female samples in true couples (RvalueC) and non-family male-female pairs (RvalueR) in the 778 genes at empirical p-values $<0.05$. (d) Differences between male and female samples in gene expression. For genes with couple effect detected at $5 \%$ empirical p-value, the average differences between male and female samples from simulated (Y-axis) were compared with the male-female differences from true couples (X-axis).

a

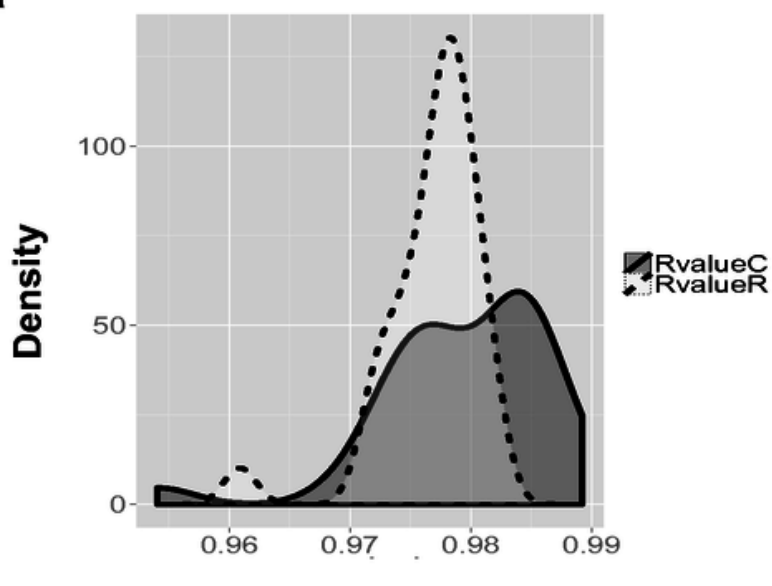

Correlation

C

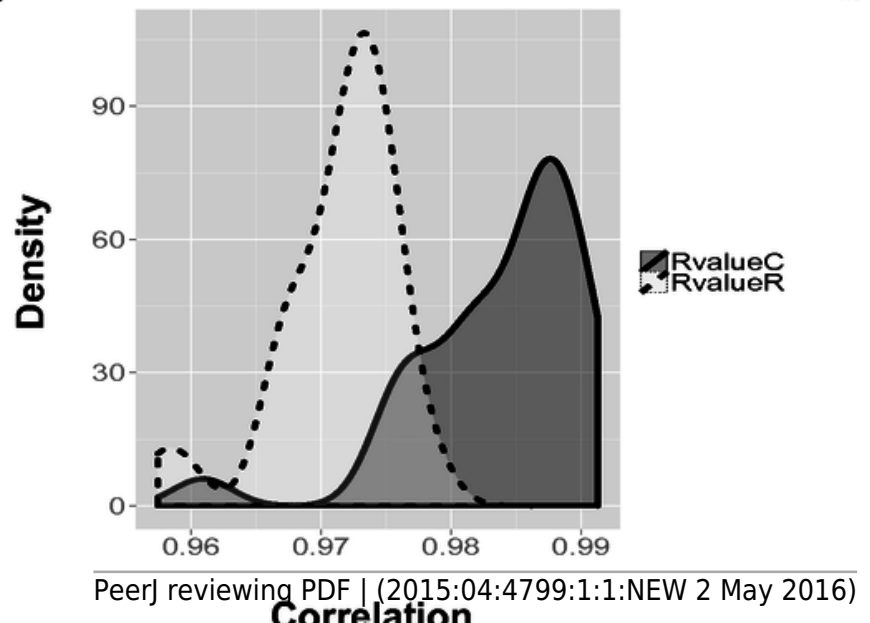

Correlation

d b
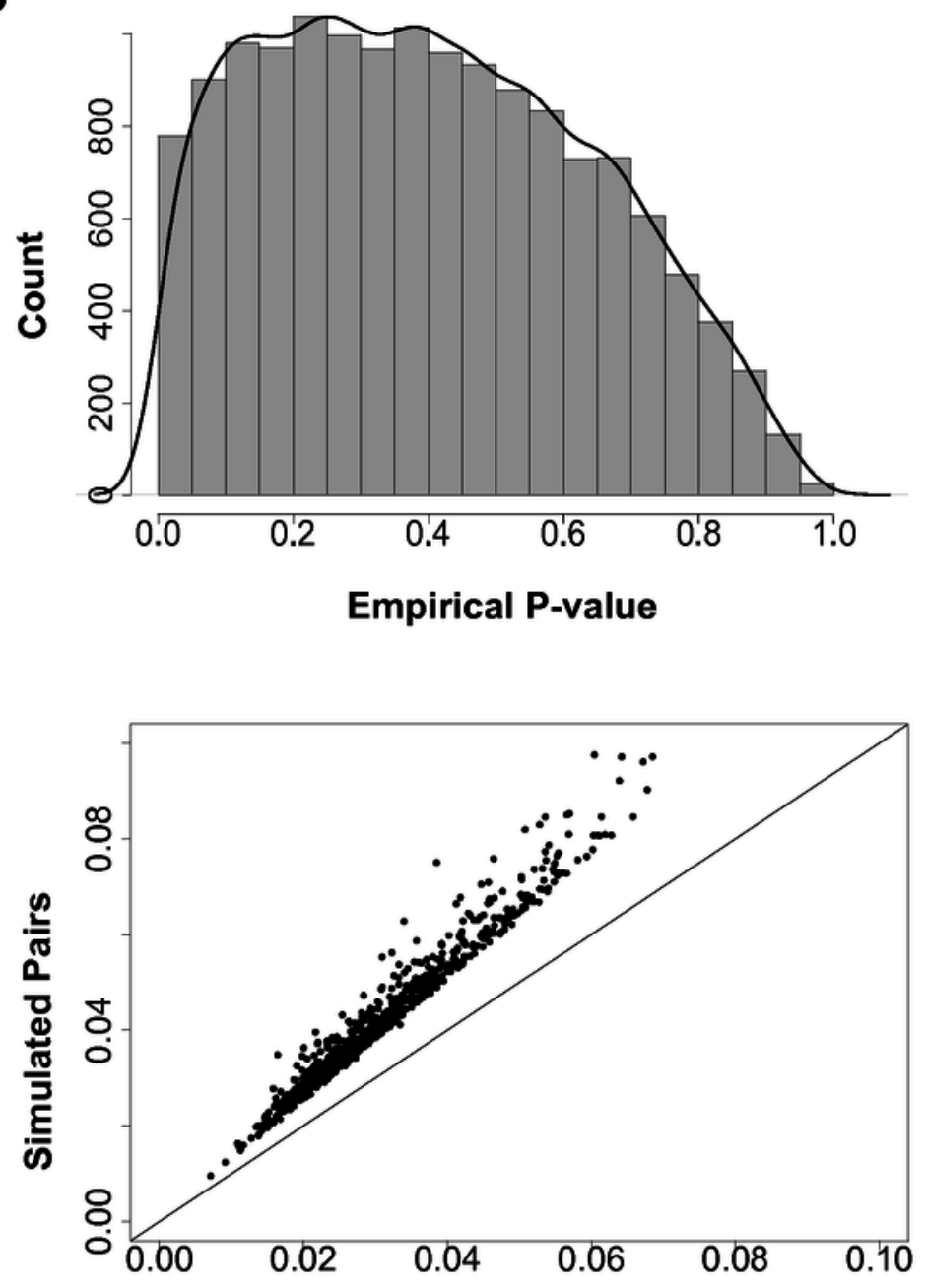

Couples 
3

Examples showing that cytosine modifications account for the 'couple effect' of gene expression.

(a) Gene expression level of DPYSL2 (encoding dihydropyrimidinase-like 2) is significantly associated with a gene-body CpG site cg24811472 ( $p=1.06 \mathrm{E}-05$ ). (b) Gene expression level of ARNT (encoding aryl hydrocarbon receptor nuclear translocator) is significantly associated with a gene-body CpG site cg19750321 ( $p=0.001)$.

a

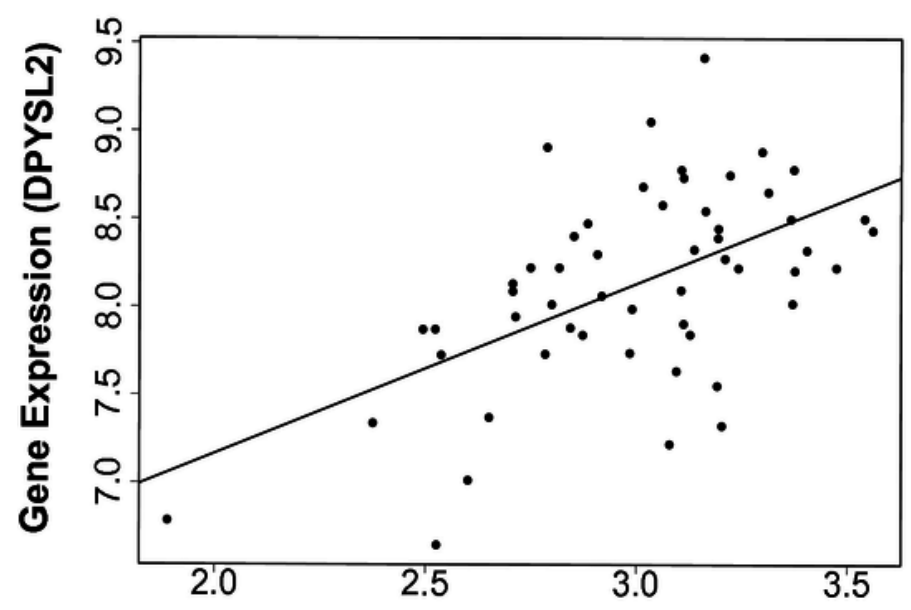

Modification Level M-value (cg24811472)

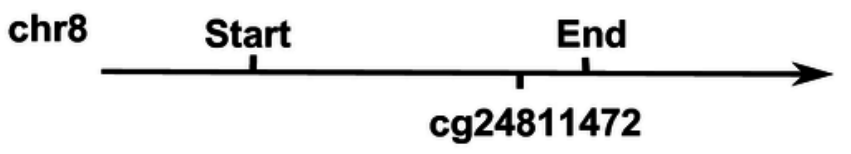

b

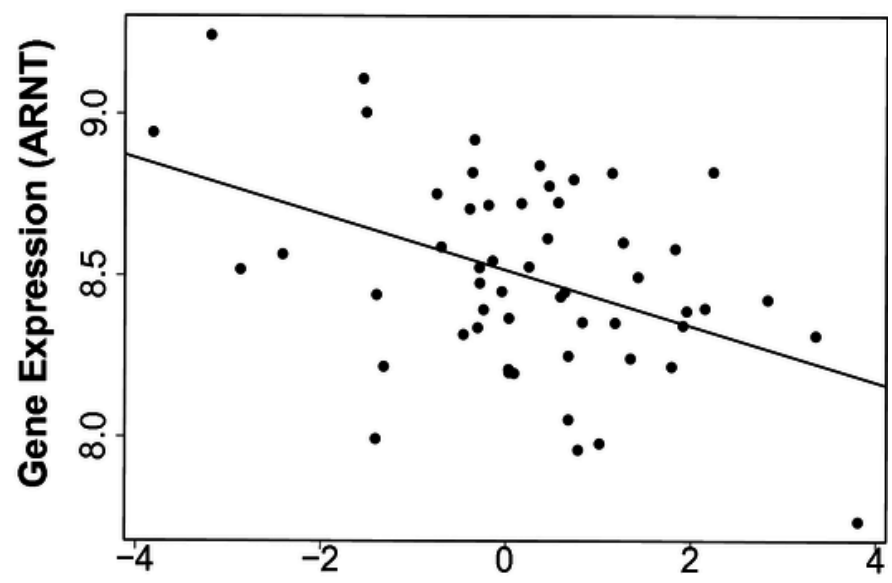

Modification Level M-value (cg19750321)

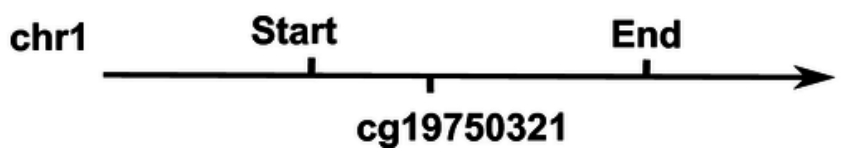




\section{Table $\mathbf{1}$ (on next page)}

CpG-Gene expression associations at q-value $<0.05$ between genes with the 'couple effect' and their local CpGs. 
1 Table 1. CpG-Gene expression associations at q-value<0.05 between genes with the 'couple effect' and 2 their local CpGs.

3

\begin{tabular}{|l|l|r|r|r|l|r|}
\hline CpGID & Gene Symbol & \multicolumn{1}{l|}{$\begin{array}{l}\text { Affymetrix } \\
\text { ID }\end{array}$} & CpGvalue & p-value & q-value & $r$ \\
\hline cg02152034 & EMID1 & 3941848 & -0.3732414 & $1.42 \mathrm{E}-06$ & 0.0227167 & -0.602693 \\
\hline cg10527635 & SNRK & 2619666 & -0.4224054 & $6.71 \mathrm{E}-06$ & 0.04227625 & -0.570362 \\
\hline cg12001078 & MAPKSP1 & 2779408 & -0.2527613 & $8.37 \mathrm{E}-06$ & 0.04227625 & -0.565482 \\
\hline cg24811472 & DPYSL2 & 3091077 & 0.9650537 & $1.06 \mathrm{E}-05$ & 0.04227625 & 0.560246 \\
\hline cg17759595 & DPYSL2 & 3091077 & 0.5309326 & $1.33 \mathrm{E}-05$ & 0.04262027 & 0.554953 \\
\hline cg04081402 & TCN2 & 3942472 & -0.5538604 & $1.70 \mathrm{E}-05$ & 0.04535223 & -0.549258 \\
\hline cg06545761 & ZCCHC14 & 3703665 & 0.0759574 & $1.99 \mathrm{E}-05$ & 0.04554208 & 0.545513 \\
\hline
\end{tabular}

4 